\title{
Statistical Angular Resolution Limit for Ultrawideband MIMO Noise Radar
}

\author{
Xiaoli Zhou, Hongqiang Wang, Yongqiang Cheng, Yuliang Qin, and Haowen Chen
}

School of Electronic Science and Engineering, National University of Defense Technology, Changsha 410073, China

Correspondence should be addressed to Hongqiang Wang; oliverwhq1970@gmail.com

Received 23 April 2014; Accepted 15 October 2014

Academic Editor: Michelangelo Villano

Copyright (C) 2015 Xiaoli Zhou et al. This is an open access article distributed under the Creative Commons Attribution License, which permits unrestricted use, distribution, and reproduction in any medium, provided the original work is properly cited.

\begin{abstract}
The two-dimensional angular resolution limit (ARL) of elevation and azimuth for MIMO radar with ultrawideband (UWB) noise waveforms is investigated using statistical resolution theory. First, the signal model of monostatic UWB MIMO noise radar is established in a 3D reference frame. Then, the statistical angular resolution limits (SARLs) of two closely spaced targets are derived using the detection-theoretic and estimation-theoretic approaches, respectively. The detection-theoretic approach is based on the generalized likelihood ratio test (GLRT) with given probabilities of false alarm and detection, while the estimation-theoretic approach is based on Smith's criterion which involves the Cramér-Rao lower bound (CRLB). Furthermore, the relationship between the two approaches is presented, and the factors affecting the SARL, that is, detection parameters, transmit waveforms, array geometry, signal-to-noise ratio (SNR), and parameters of target (i.e., radar cross section (RCS) and direction), are analyzed. Compared with the conventional radar resolution theory defined by the ambiguity function, the SARL reflects the practical resolution ability of radar and can provide an optimization criterion for radar system design.
\end{abstract}

\section{Introduction}

As a novel class of radar system, multiple-input multipleoutput (MIMO) radar has gained popularity and attracted attention [1]. MIMO radar can offer additional degrees of freedom by employing multiple transmit and receive antennas, and it possess significant potentials for fading mitigation, resolution enhancement, and interference, and jamming suppression. Fully exploiting these potentials can improve target detection, parameter estimation, and target tracking and recognition performance [2]. MIMO radar uses multiple antennas to simultaneously transmit noncoherent waveforms, while the ultrawideband (UWB) noise waveform is an ideal candidate for MIMO operation [3]. Recently, there has been some active research on using UWB noise radar in MIMO configuration [3-5]. A UWB noise radar, transmitting a noise or noise-like waveform with a fractional bandwidth of over $25 \%$ or an absolute bandwidth of over $500 \mathrm{MHz}$ [3], has high range resolution, uncoupled ambiguity function $(\mathrm{AF})$, low probabilities of interception and detection, and immunity from interference $[5,6]$. Combing the benefits of MIMO radar and UWB noise radar, UWB MIMO noise radar satisfies some important military requirements and is considered to be a promising technique for high resolution target detection and estimation [4].

For UWB MIMO noise radar, a typical function is target localization [2,7], and there exists performance improvement in this application, while it is necessary to know the performance level of target localization expected from a particular radar configuration, and resolution limit is an important performance measure indicating the capability to resolve closely spaced targets. Among the tools characterizing the resolution limit, the classic AF is commonly used to describe the inherent resolution properties of time delay and Doppler for a specific waveform [8]. Early, AF concentrated on the monostatic narrowband applications. Owing to the potential advantages of the UWB waveform and MIMO radar system, wideband/UWB AF $[6,9,10]$ and MIMO AF $[10-12]$ are then investigated.

Although $\mathrm{AF}$ describes the resolving ability of radar waveform and MIMO AF can simultaneously characterize the effects of array geometry, transmitted waveform, and target scattering on resolution performance [2], the effect of environment noise is neglected. Moreover, the conventional 
resolution limit (i.e., AF) is limited by the Rayleigh limit, whereas the super-resolution theory indicates that resolution beyond the Rayleigh limit is possible under certain conditions related to signal-to-noise ratio (SNR).

The statistical resolution limit (SRL) [13], deduced from the statistical characteristic of radar measurement and where the uncertainty of measurement is taken into account, has attracted much attention recently. The SRL can be used to analyze the statistical resolution capability beyond the Rayleigh's criterion and reflect the practical resolution ability of radar. There are mainly three approaches to define the SRL. The earliest approach is based on the mean null spectrum [14] which is quite simple, whereas its application is limited to the specific estimation algorithm. In practical applications, there exist another two commonly used approaches, independent of the algorithm, to define the SRL. (I) One is based on detection theory; the resolution problem is modeled as a hypothesis test to decide if the two sources are resolvable. And the resolution limit is defined by the probability of error or right criterion [15-21]. (II) The other is based on estimation accuracy; the SRL is given with the Cramér-Rao lower bound (CRLB) [13, 15, 16, 22-24]. There are two main criteria based on the CRLB: Lee's criterion [25] and Smith's criterion [13].

In recent years, the SRLs of DOD (direction of departure) and DOA (direction of arrival) for MIMO radar have been deeply researched [21, 24], whereas the majority of this published work has assumed narrowband signal scenarios, which cannot be applied to the wideband/UWB situation directly. The SRL of wideband/UWB MIMO radar has not been investigated, not to mention UWB MIMO noise radar.

In this paper, the $2 \mathrm{D}$ statistical angular resolution limits (SARLs) of elevation and azimuth for UWB MIMO noise radar in a 3D reference frame are derived. The prespecified detection-theoretic and estimation-theoretic approaches are used in the derivations, which are based on GLRT with the constraints on probabilities of false alarm and detection, and CRLB with the constraint on Smith's criterion, respectively. The closed-form expressions of SARL are achieved, which are compact and can provide useful qualitative information on the behavior of resolution limit. Moreover, the relationships between SARL and different factors (i.e., SNR, detection parameters, array geometry, and the radar cross section (RCS) and direction of target) are analyzed. As for the application, the SARL presented in this paper can be used to evaluate the performance of sensor arrays in target localization (such as in radar and sonar), and the approaches may be applied to general detection and parameter estimation problems.

This paper is organized as follows. In Section 2, we introduce the signal model and the related assumptions which are used in the derivations. In Section 3, detectiontheoretic approach is presented to investigate the SARL. Then the SARL is further derived based on CRLB with the Smith's criterion, and the relationship between the two approaches is discussed in Section 4. In Section 5, the results obtained in Sections 3 and 4 are discussed to provide further insight into the SARL, and some insightful properties revealed by the results of simulations are given. Finally, some comments and conclusions are presented in Section 6.
A comment on notation is as follows: we use boldface lowercase letters for vectors and boldface uppercase letters for matrices. $(\cdot)^{H},(\cdot)^{*},(\cdot)^{T}$, and $(\cdot)^{-1}$ denote the conjugate transpose, conjugate, transpose, and inverse of a matrix, respectively. $E\{\cdot\}$ is used for expectation with respect to the random variable. $\mathfrak{R}\{\cdot\}$ and $\mathfrak{\Im}\{\cdot\}$ denote the real and imaginary parts, respectively. Finally, $\|\cdot\|$ denotes the Frobenius norm of a vector and $\mathbf{I}_{N}$ is the $N$ th-order identity matrix.

\section{Problem Setup}

2.1. Assumptions. Throughout the paper, it is assumed that the following assumptions hold.

(1) The radar is operated in monostatic configuration and then the DOD is approximately equal to the DOA.

(2) The targets are point targets located in the far-field of the radar array.

(3) The background noise is an independent additive wide-sense stationary (WSS) complex circular white Gaussian random process with zero-mean and variance $\sigma_{n}^{2}$.

(4) All transmit waveforms are mutually orthogonal band-limited noise waveforms with equal power $\sigma_{s}^{2}$ and bandwidth $B_{w}$. Meanwhile, the signal spectral components at different frequencies are also mutually orthogonal.

(5) The channel attenuation is ignored.

(6) The coherent processing time for signal and noise is sufficient.

2.2. Signal Model. Consider two closely spaced targets in the far-field for MIMO radar. Target T1 is assumed as the reference target with a known direction $\boldsymbol{\theta}_{1}=\left(\varphi_{1}, \phi_{1}\right)$ and RCS, while target T2, whose direction $\boldsymbol{\theta}_{2}=\left(\varphi_{2}, \phi_{2}\right)$ is unknown, is close to T1. $\varphi_{i} \in[0, \pi / 2]$ and $\phi_{i} \in[0,2 \pi]$ denote the elevation and azimuth of the $i$ th target $(\forall i \in\{1,2\})$, respectively.

The MIMO radar geometry is shown in Figure 1. Both the $N$-element transmitters and $M$-element receivers, which are located in the same plane, are circular arrays (but not limited to circular array) with radii $r_{t}$ and $r_{r}$, respectively. The origin $O$ of the Cartesian coordinate system is set up as the center of the arrays, wherein the radar array and targets are considered as point objects.

In this paper, a single snapshot is considered. Then the demodulated baseband signal received by the $m$ th receiver can be expressed as [7]:

$$
y_{m}(t)=\sum_{i=1}^{2} \alpha_{i} \sum_{n=1}^{N} s_{n}\left(t-\tau_{m n}^{i}\right) e^{-j 2 \pi f_{c} \tau_{m n}^{i}}+w_{m}(t),
$$

where $s_{n}(t)$ is the baseband stochastic signal transmitted by the $n$th transmitter with the bandwidth $B_{w}, w_{m}$ is the noise received by the $m$ th receiver, and $f_{c}$ is the carrier frequency of the transmit waveforms. $\alpha_{i}$ denotes the complex amplitude proportional to the RCS of the $i$ th target. 


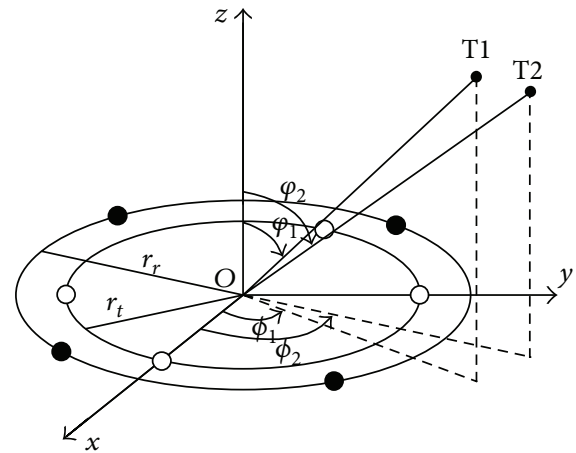

- Receiver

$\bigcirc$ Transmitter

FIGURE 1: Geometry of monostatic MIMO radar with circular arrays.

$\tau_{m n}^{i}=\sin \varphi_{i}\left(r_{r} \cos \left(\phi_{i}-\vartheta_{r m}\right)+r_{t} \cos \left(\phi_{i}-\vartheta_{t n}\right)\right) / c$ is the relative propagation delay corresponding to the $n$th transmitter (located at $\boldsymbol{\theta}_{t n}=\left(\pi / 2, \vartheta_{t n}\right)$ ) and the $m$ th receiver (located at $\left.\boldsymbol{\theta}_{r m}=\left(\pi / 2, \vartheta_{r m}\right)\right)$ with respect to the reference point $O$, where $c$ is the speed of wave propagation.

Since $s_{n}(t)$ is an UWB stochastic signal which is not convenient to analysis, thus $s_{n}(t)$ is expressed as the spectral representation $s_{n}(t)=\int_{f} e^{j 2 \pi f t} d S_{n}(f)$ in the frequency domain. $d S_{n}(f)$ denotes a measure of the signal spectrum at frequency $f$, which is independent of variable $t$ and will simplify the derivations involving the stochastic signal.

\section{SARL Based on Detection-Theoretic Approach}

In this section, the 2D SARL, based on the hypothesis test (more precisely, GLRT), is derived using the signal model in Section 2.

3.1. Statistic of the Observation. From the statistical signal processing theory we know that the sufficient statistic is the effective summarization and makes the probability density function (PDF) be independent with the unknown parameters. For UWB MIMO noise radar, one of the sufficient statistics for targets resolving is

$$
\mathbf{E} \triangleq \mathbf{y}(t)=\mathbf{x}(t)+\mathbf{w}(t)
$$

where $\mathbf{y}(t)=\left[y_{1}(t), \ldots, y_{M}(t)\right]^{T}, \mathbf{x}(t)=\left[x_{1}(t), \ldots, x_{M}(t)\right]^{T}$, $\mathbf{w}(t)=\left[w_{1}(t), \ldots, w_{M}(t)\right]^{T}, x_{m}(t)=\sum_{i=1}^{2} \alpha_{i} \sum_{n=1}^{N} s_{n}(t-$ $\left.\tau_{m n}^{i}\right) e^{-j 2 \pi f_{c} \tau_{m n}^{i}}$.

3.2. Linear Form of the Signal Model. The 2D SARL can be expressed as $\boldsymbol{\delta} \triangleq\left[\begin{array}{ll}\delta_{\varphi} & \delta_{\phi}\end{array}\right]^{T}$, where $\delta_{\varphi} \triangleq \varphi_{2}-\varphi_{1}$ and $\delta_{\phi} \triangleq \phi_{2}-\phi_{1}$. The derivation of $\delta_{\varphi}$ and $\delta_{\phi}$ is a nonlinear optimization problem which is analytically intractable. We therefore approximate the model to be linear w.r.t. the unknown parameters.
Substituting $s_{n}(t)=\int_{f} e^{j 2 \pi f t} d S_{n}(f)$ into $x_{m}(t)$, the first order Taylor expansion of $x_{m}(t)$ around $\boldsymbol{\delta}=\mathbf{0}$ is given as

$$
\begin{gathered}
x_{m}(t) \approx\left(\alpha_{1}+\alpha_{2}\right) \sum_{n=1}^{N} \int_{f} e^{j 2 \pi\left(f\left(t-\tau_{m n}^{1}\right)-f_{c} \tau_{m n}^{1}\right)} d S_{n}(f) \\
-j 2 \pi \alpha_{2} \delta_{\varphi} \cos \varphi_{1} \sum_{n=1}^{N} \int_{f} a_{m n}\left(f+f_{c}\right) \\
\quad \times e^{j 2 \pi\left(f\left(t-\tau_{m n}^{1}\right)-f_{c} \tau_{m n}^{1}\right)} d S_{n}(f) \\
+j 2 \pi \alpha_{2} \delta_{\phi} \sin \varphi_{1} \sum_{n=1}^{N} \int_{f} b_{m n}\left(f+f_{c}\right) \\
\times e^{j 2 \pi\left(f\left(t-\tau_{m n}^{1}\right)-f_{c} \tau_{m n}^{1}\right)} d S_{n}(f) .
\end{gathered}
$$

Then, the sufficient statistic can be expressed as

$$
\begin{gathered}
\mathbf{y}=\mathbf{G} \boldsymbol{\zeta}+\mathbf{w}, \\
\zeta=\left[\begin{array}{ll}
\alpha_{1}+\alpha_{2} & \alpha_{2} \boldsymbol{\delta}^{T}
\end{array}\right]^{T}, \\
\mathbf{G}=\left[\begin{array}{lll}
\mathbf{B} & \mathbf{C} & \mathbf{D}
\end{array}\right], \\
B_{m}=\sum_{n=1}^{N} \int_{f} e^{j 2 \pi\left(f\left(t-\tau_{m n}^{1}\right)-f_{c} \tau_{m n}^{1}\right)} d S_{n}(f), \\
C_{m}=-j 2 \pi \cos \varphi_{1} \sum_{n=1}^{N} \int_{f} a_{m n}\left(f+f_{c}\right) \\
D_{m}=j 2 \pi \cos \varphi_{1} \sum_{n=1}^{N} \int_{f} b_{m n}\left(f+f_{c}\right) \\
\times e^{j 2 \pi\left(f\left(f\left(t-\tau_{m n}^{1}\right)-f_{c} \tau_{m n}^{1}\right)\right.} d S_{n}(f),
\end{gathered}
$$

where $B_{m}, C_{m}$, and $D_{m}$ represent the $m$ th element of $\mathbf{B}, \mathbf{C}$, and $\mathrm{D}$, respectively. $a_{m n}$ and $b_{m n}$ are denoted by

$$
\begin{aligned}
& a_{m n}=\frac{\left(r_{r} \cos \left(\phi_{1}-\vartheta_{r m}\right)+r_{t} \cos \left(\phi_{1}-\vartheta_{t n}\right)\right)}{c}, \\
& b_{m n}=\frac{\left(r_{r} \sin \left(\phi_{1}-\vartheta_{r m}\right)+r_{t} \sin \left(\phi_{1}-\vartheta_{t n}\right)\right)}{c} .
\end{aligned}
$$

3.3. Derivation of the $S A R L$. It is a common sense that the targets can be resolved easily and the probability of detection is higher if the spacing between the two targets is larger. In this sense, the probability of detection can be used to represent the resolvability of two closely spaced targets. Consequently, the resolution problem can be modeled as a hypothesis test problem, and the relationships between the minimal resolvable $\delta$ and the factors affecting the resolution limit can be obtained. Denoting that $\mathbf{P}=\left[\mathbf{0}, \mathbf{I}_{2}\right]$, as $\mathbf{P} \boldsymbol{\zeta}=\alpha_{2} \boldsymbol{\delta}$ 
and $\alpha_{2} \neq 0$, the corresponding hypothesis for the resolution problem is given by

$$
\begin{array}{ll}
\mathscr{H}_{0}: & \mathbf{P} \zeta=\mathbf{0} \\
\mathscr{H}_{1}: & \mathbf{P} \zeta \neq \mathbf{0},
\end{array}
$$

where $\mathscr{H}_{1}$ and $\mathscr{H}_{0}$ indicate that the two targets are resolvable or not, respectively.

A basic method for hypothesis test is the classical approach based on Neyman-Pearson (NP) lemma [26]. According to the NP lemma, the optimum solution to the above hypothesis test problem is the likelihood ratio test (LRT). However, since $\boldsymbol{\delta}$ is unknown, it is impossible to give the PDF exactly under $\mathscr{H}_{1}$ and design an optimal detector in the NP sense. A common approach is the GLRT in this case. GLRT first computes the maximum likelihood estimates (MLEs) of the unknown parameters and then uses the estimated values to form the standard NP detector. Although GLRT is not optimal, it is an asymptotically uniformly most powerful (UMP) test among all the invariant statistical tests [26], and its performance is very close to that of an ideal detector, to which the values of all the parameters in the model are known. Hence, the performance of the described detector can be reasonably considered as an approximate performance bound in practice. According to (7), the expression of GLRT is

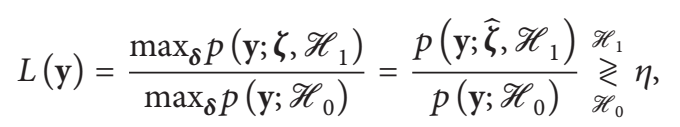

where $p\left(\mathbf{y} ; \zeta, \mathscr{H}_{1}\right)$ and $p\left(\mathbf{y} ; \mathscr{H}_{0}\right)$ are the PDFs under $\mathscr{H}_{1}$ and $\mathscr{H}_{0}$, respectively. $\eta$ is detection threshold. $\widehat{\zeta}=\left(\mathbf{G}^{\mathrm{H}} \mathbf{G}\right)^{-1} \mathbf{G}^{\mathrm{H}} \mathbf{y}$ is the MLE of $\boldsymbol{\xi}$ when $p\left(\mathbf{y} ; \widehat{\zeta}, \mathscr{H}_{1}\right)$ is maximized. Equivalently, the GLRT in (8) can be rewritten as

$$
T(\mathbf{y})=2 \ln L(\mathbf{y}) \underset{\mathscr{H}_{0}}{\stackrel{\mathscr{H}_{1}}{\gtrless}} \eta^{\prime}=2 \ln \eta .
$$

Then the GLRT statistic for the approximated model yields [26]

$$
T(\mathbf{y})=\frac{2}{\sigma_{n}^{2}}(\mathbf{P} \widehat{\zeta})^{\mathbf{H}}\left(\mathbf{P}\left(\mathbf{G}^{\mathrm{H}} \mathbf{G}\right)^{-1} \mathbf{P}^{\mathrm{H}}\right)^{-1} \mathbf{P} \widehat{\zeta} .
$$

To choose the value of $\eta^{\prime}$, the asymptotic performance of the test [26] is formed as (11), as the expression of GLRT may be generally complicated and its performance may be difficult to analyze. Consider

$$
T(\mathbf{y}) \sim \begin{cases}\chi_{2 M}^{2}, & \mathscr{H}_{0} \\ \chi_{2 M}^{\prime 2}\left(\lambda\left(p_{f a}, p_{d}\right)\right), & \mathscr{H}_{1},\end{cases}
$$

where $\chi_{2 M}^{2}$ and $\chi_{2 M}^{\prime 2}\left(\lambda\left(p_{f a}, p_{d}\right)\right)$ are the central and noncentral chi-square distribution with $2 M$ degrees of freedom, respectively. The noncentrality parameter is $\lambda\left(p_{f a}, p_{d}\right)$ which is critical in illuminating the relationship between the SNR and SARL. Then $\eta^{\prime}$ is conditioned by the probabilities of false alarm and detection, which are defined as $p_{f a}=$ $Q_{\chi_{2 M}^{2}}\left(\eta^{\prime}\right)$ and $p_{d}=Q_{\chi_{2 M}^{\prime 2}\left(\lambda\left(p_{f a}, p_{d}\right)\right)}\left(\eta^{\prime}\right)$, respectively. $Q_{\chi_{2 M}^{2}}(\cdot)$ and $Q_{\chi_{2 M}^{\prime 2}\left(\lambda\left(p_{f a}, p_{d}\right)\right)}(\cdot)$ denote the right tail probabilities of $\chi_{2 M}^{2}$ and $\chi_{2 M}^{\prime 2}\left(\lambda\left(p_{f a}, p_{d}\right)\right)$, respectively. Then $\lambda\left(p_{f a}, p_{d}\right)$ can be computed as the solution of

$$
Q_{\chi_{2 M}^{2}}^{-1}\left(p_{f a}\right)=Q_{\chi_{2 M}^{2}\left(\lambda\left(p_{f a}, p_{d}\right)\right)}^{-1}\left(p_{d}\right) .
$$

On the other hand, $\lambda\left(p_{f a}, p_{d}\right)$ can be given as

$$
\lambda\left(p_{f a}, p_{d}\right)=\frac{2}{\sigma_{n}^{2}}(\mathbf{P} \zeta)^{\mathbf{H}}\left(\mathbf{P}\left(\mathbf{G}^{\mathbf{H}} \mathbf{G}\right)^{-1} \mathbf{P}^{\mathbf{H}}\right)^{-1}(\mathbf{P} \zeta) .
$$

From the viewpoint of statistics, it is impossible to resolve the two closely spaced targets at 100 percent, whereas they are resolvable at a given probability. Since the constraints on $p_{f a}$ and $p_{d}$ for test (7) are assigned, the SRL can be rigorously defined by the constraints; otherwise, the resolution limit can be arbitrarily low and the result may be meaningless [20]. Based on the constraints, we may claim that the two targets are statistically resolved under the constraints on $p_{f a}$ and $p_{d}$. In other words, the probability to resolve both targets is $p_{d}$ for a given $p_{f a}$. There definitely exists a minimum $\boldsymbol{\delta}$ so that both constraints can be satisfied. If $\boldsymbol{\delta}$ is lower than the minimum value, the constraints on $p_{f a}$ and $p_{d}$ will be broken. The minimum $\boldsymbol{\delta}$, therefore, can be taken as the SRL.

When computing $\left(\mathbf{P}\left(\mathbf{G}^{\mathrm{H}} \mathbf{G}\right)^{-1} \mathbf{P}^{\mathrm{H}}\right)^{-1}$, it is necessary to obtain $\mathbf{B}^{\mathrm{H}} \mathbf{B}, \mathbf{B}^{\mathrm{H}} \mathbf{C}, \mathbf{B}^{\mathrm{H}} \mathbf{D}, \mathbf{C}^{\mathrm{H}} \mathbf{C}, \mathbf{C}^{\mathrm{H}} \mathbf{D}$, and $\mathbf{D}^{\mathrm{H}} \mathbf{D}$. Since $s_{n}(t)$ is a stochastic signal, the expectation operator is applied to calculate $\left(\mathbf{P}\left(\mathbf{G}^{\mathrm{H}} \mathbf{G}\right)^{-1} \mathbf{P}^{\mathrm{H}}\right)^{-1}$. As we have assumed that the spectral components at different frequencies are mutually orthogonal in Section 2, then [27]

$$
E\left[d S_{n}(f) d S_{n}^{*}\left(f^{\prime}\right)\right]= \begin{cases}\rho_{n}(f) d f, & f=f^{\prime} \\ 0, & f \neq f^{\prime},\end{cases}
$$

where $\rho_{n}(f)$ is the power spectrum density (PSD) of $s_{n}(t)$ at the frequency $f$. From (5) and (14), we can obtain

$$
E\left\{\mathbf{B}^{\mathrm{H}} \mathbf{B}\right\}=M N \sigma_{s}^{2},
$$

where $\sigma_{s}^{2}=\int_{f} \rho_{n}(f) d f$ is the power of each transmitter. The same way is used to obtain $E\left\{\mathbf{B}^{\mathrm{H}} \mathbf{C}\right\}, E\left\{\mathbf{B}^{\mathrm{H}} \mathbf{D}\right\}$ and so on.

The total SNR is defined as SNR $=\left(N \sigma_{s}^{2}\right) /\left(M \sigma_{n}^{2}\right)$, where $\sigma_{n}^{2}$ can be obtained from (13). Finally, the relationship between the SARL $\boldsymbol{\delta}$ and the minimum SNR, which is required to resolve two closely spaced targets, is given by

$$
\mathrm{SNR}=\frac{N \lambda\left(p_{f a}, p_{d}\right)}{8 \pi^{2} M\left\|\alpha_{2}\right\|^{2} \boldsymbol{\delta}^{T}\left(f_{c}^{2}\left(\boldsymbol{\varepsilon}_{1}-\boldsymbol{\varepsilon}_{2} / M N\right)+B_{\mathrm{rms}}^{2} \boldsymbol{\varepsilon}_{1}\right) \boldsymbol{\delta}},
$$

where $B_{\mathrm{rms}}=\sqrt{\int_{f} f^{2} \rho(f) d f / \int_{f} \rho(f) d f}$ is the root mean square (RMS) bandwidth. The RMS bandwidth, which is not equivalent to bandwidth $B_{w}$, is commonly used as a basic 
quantitative characteristic to describe the feature of power spectra. Consider

$$
\begin{gathered}
\boldsymbol{\varepsilon}_{1}=\left[\begin{array}{cc}
\left(\cos \varphi_{1}\right)^{2} \varepsilon_{1} & -\sin \varphi_{1} \cos \varphi_{1} \varepsilon_{3} \\
-\sin \varphi_{1} \cos \varphi_{1} \varepsilon_{3} & \left(\sin \varphi_{1}\right)^{2} \varepsilon_{2}
\end{array}\right], \\
\boldsymbol{\varepsilon}_{2}=\left[\begin{array}{cc}
\left(\cos \varphi_{1}\right)^{2} \varepsilon_{4}^{2} & -\sin \varphi_{1} \cos \varphi_{1} \varepsilon_{4} \varepsilon_{5} \\
-\sin \varphi_{1} \cos \varphi_{1} \varepsilon_{4} \varepsilon_{5} & \left(\sin \varphi_{1}\right)^{2} \varepsilon_{5}^{2}
\end{array}\right], \\
\varepsilon_{1}=\sum_{m=1}^{M} \sum_{n=1}^{N} a_{m n}^{2}, \quad \varepsilon_{2}=\sum_{m=1}^{M} \sum_{n=1}^{N} b_{m n}^{2}, \quad \varepsilon_{3}=\sum_{m=1}^{M} \sum_{n=1}^{N} a_{m n} b_{m n}, \\
\varepsilon_{4}=\sum_{m=1}^{M} \sum_{n=1}^{N} a_{m n}, \quad \varepsilon_{5}=\sum_{m=1}^{M} \sum_{n=1}^{N} b_{m n} .
\end{gathered}
$$

From (16), the SARL depends not only on SNR, but also on the detection parameters, transmit waveforms, array geometry, RCS, and direction of target. In Particular, $\varepsilon_{1}=$ $\varepsilon_{2}=M N\left(r_{t}^{2}+r_{r}^{2}\right) /\left(2 c^{2}\right)$ and $\varepsilon_{3}=\varepsilon_{4}=\varepsilon_{5}=0$ for uniform circular array (UCA) when $M, N \neq 1$. Then the SARL can be given by

$$
\begin{aligned}
\cos ^{2} \varphi_{1} \delta_{\varphi}^{2}+\sin ^{2} \varphi_{1} \delta_{\phi}^{2} & \\
& =\frac{c^{2} \lambda\left(p_{f a}, p_{d}\right)}{4 \pi^{2} M^{2}\left\|\alpha_{2}\right\|^{2}\left(r_{t}^{2}+r_{r}^{2}\right)\left(f_{c}^{2}+B_{\mathrm{rms}}^{2}\right) \mathrm{SNR}} .
\end{aligned}
$$

Then we can conclude that the SARL is independent of the azimuth of target for UCA.

To complement the results in this section, we carry out the Fisher information matrix (FIM) derivation for the general signal model to achieve the SARL expression form the parameter estimation perspective in the following.

\section{SARL Based on Estimation-Theoretic Approach}

In this section, we derive and analyze the SARL for UWB MIMO noise radar, based on the Smith's criterion which involves the CRLB. The SARL can be defined as the solution of the Smith equation $\delta=\sqrt{\operatorname{CRLB}(\delta)}$. To solve the equation, we need to have a closed-form expression of $\operatorname{CRLB}(\delta)$. The derivation process is as follows. First, the FIM derivation for the general signal model is carried out. Then, the closedform expression of the CRLB for the considered problem is achieved. Finally, we derive in closed form the analytic SARL under the assumption of UCA.

4.1. FIM Derivation. Since T1 is considered as the reference target, $\alpha_{1}, \varphi_{1}$, and $\phi_{1}$ are assumed to be known. Thus, the vector of unknown parameters in (2) is

$$
\boldsymbol{\xi}=\left[\begin{array}{lll}
\underbrace{\alpha_{2}^{R}}_{\boldsymbol{\alpha}} \alpha_{2}^{I} & \underbrace{\delta_{\varphi}}_{\boldsymbol{\delta}} \delta_{\phi}
\end{array}\right]^{T},
$$

where $\alpha_{2}^{R}=\mathfrak{R}\left\{\alpha_{2}\right\}$ and $\alpha_{2}^{I}=\mathfrak{\Im}\left\{\alpha_{2}\right\}$ denote the real and imaginary parts of $\alpha_{2}$; that is, $\alpha_{2}=\alpha_{2}^{R}+j \alpha_{2}^{I}$. In (19), we identify two sets of parameters: parameters of interest $\delta \triangleq$ $\left[\begin{array}{ll}\delta_{\varphi} & \delta_{\phi}\end{array}\right]^{T}$ and nuisance parameters $\boldsymbol{\alpha} \triangleq\left[\begin{array}{ll}\alpha_{2}^{R} & \alpha_{2}^{I}\end{array}\right]^{T}$.

The FIM $\mathbf{J}(\xi)$ with respect to the vector $\xi$ is given by [7]

$$
\mathbf{J}(\boldsymbol{\xi})=E_{\mathbf{y} \mid \xi}\left\{\left[\frac{\partial}{\partial \xi} \log p(\mathbf{y} \mid \boldsymbol{\xi})\right]\left[\frac{\partial}{\partial \xi} \log p(\mathbf{y} \mid \boldsymbol{\xi})\right]^{H}\right\},
$$

where $p(\mathbf{y} \mid \boldsymbol{\xi})$ is the joint PDF of $\mathbf{y}$ conditioned on $\boldsymbol{\xi}$ and $E_{\mathrm{y} \mid \xi}\{\cdot\}$ is the conditional expectation of $\mathbf{y}$ given $\xi$.

The received signals $\mathbf{y}$ are parameterized by the unknown parameters $\xi$. The conditional joint PDF of the observations at the receivers, given by (1), is

$$
p(\mathbf{y} \mid \xi) \propto \exp \left\{-\frac{1}{\sigma_{n}^{2}} \sum_{m=1}^{M} \int_{T}\left\|y_{m}(t)-x_{m}(t)\right\|^{2} d t\right\} .
$$

Then we develop the FIM for $\xi$, based on the conditional PDF in (21). The expression for (20) is obtained using

$$
\begin{array}{r}
{[\mathbf{J}(\boldsymbol{\xi})]_{i j}=[\mathbf{J}(\xi)]_{j i}=-E_{\mathbf{y} \mid \xi}\left\{\frac{\partial^{2} \log p(\mathbf{y} \mid \boldsymbol{\xi})}{\partial \boldsymbol{\xi}_{i} \partial \boldsymbol{\xi}_{j}}\right\}} \\
i, j=1,2,3,4 .
\end{array}
$$

Before the derivation continues, some conclusions should be made. Denote $\dot{s}_{n}\left(t-\tau_{m n}^{l}\right)=\partial s_{n}\left(t-\tau_{m n}^{l}\right) / \partial \tau_{m n}^{l}$, as described in Section $2 ; s_{n}(t)=\int_{f} e^{j 2 \pi f t} d S_{n}(f)$; then, $\dot{s}_{n}\left(t-\tau_{m n}^{l}\right)$ can be expressed as

$$
\dot{s}_{n}\left(t-\tau_{m n}^{l}\right)=\int_{f}-j 2 \pi f e^{j 2 \pi f\left(t-\tau_{m n}^{l}\right)} d S_{n}(f) .
$$

According to the conclusion described in (14), the conclusions can be drawn as follows:

$$
\begin{aligned}
& E\left\{\int_{T} \dot{s}_{n}\left(t-\tau_{m n}^{1}\right) s_{n}^{*}\left(t-\tau_{m n}^{1}\right) d t\right\}=\int_{f}-j 2 \pi f \rho_{n} d f=0, \\
& E\left\{\int_{T} \dot{s}_{n}\left(t-\tau_{m n}^{1}\right) \dot{s}_{n}^{*}\left(t-\tau_{m n}^{1}\right) d t\right\}=\int_{f}(2 \pi f)^{2} \rho_{n} d f \\
& =4 \pi^{2} B_{\mathrm{rms}}^{2} \sigma_{s}^{2} .
\end{aligned}
$$

Based on (14) and (24), the expression for the FIM $\mathbf{J}(\xi)$ is derived in the Appendix.

4.2. CRLB Derivation. The CRLB provides a lower bound for the MSE of any unbiased estimator for unknown parameters. Given a vector parameter $\boldsymbol{\xi}$, the CRLB is defined as

$$
\operatorname{CRLB}(\boldsymbol{\xi})=[\mathbf{J}(\boldsymbol{\xi})]^{-1} \text {. }
$$

To give an insight into the terms in the expression of FIM, $\mathbf{J}(\boldsymbol{\xi})$ defined by (20) can be partitioned as

$$
[\mathbf{J}(\xi)]=\left[\begin{array}{cc}
\mathbf{J}_{\alpha \alpha} & \mathbf{J}_{\alpha \delta}^{H} \\
\mathbf{J}_{\alpha \delta} & \mathbf{J}_{\delta \delta}
\end{array}\right]
$$


where the elements $\mathbf{J}_{\alpha \alpha}, \mathbf{J}_{\alpha \delta}$, and $\mathbf{J}_{\delta \delta}$ are defined as

$$
\begin{aligned}
& \mathbf{J}_{\boldsymbol{\alpha} \boldsymbol{\alpha}}=2 M N \operatorname{snr} \mathbf{I}_{2} \\
& \mathbf{J}_{\boldsymbol{\alpha} \delta}=\mathbf{J}_{\boldsymbol{\alpha} \delta}^{H}=4 \pi f_{c} \operatorname{snr}\left[\begin{array}{cc}
\alpha_{2}^{I} \cos \varphi_{1} \varepsilon_{4} & -\alpha_{2}^{R} \cos \varphi_{1} \varepsilon_{4} \\
-\alpha_{2}^{I} \sin \varphi_{1} \varepsilon_{5} & \alpha_{2}^{R} \sin \varphi_{1} \varepsilon_{5}
\end{array}\right]
\end{aligned}
$$

$$
\begin{aligned}
\mathrm{J}_{\delta \delta}=8 \pi^{2}\left\|\alpha_{2}\right\|^{2}\left(B_{\mathrm{rms}}^{2}+f_{c}^{2}\right) & \\
& \times \operatorname{snr}\left[\begin{array}{cc}
\cos ^{2} \varphi_{1} \varepsilon_{1} & -\sin \varphi_{1} \cos \varphi_{1} \varepsilon_{3} \\
-\sin \varphi_{1} \cos \varphi_{1} \varepsilon_{3} & \sin ^{2} \varphi_{1} \varepsilon_{2}
\end{array}\right],
\end{aligned}
$$

where snr $=\sigma_{s}^{2} / \sigma_{n}^{2}$ and $\mathbf{I}_{2}$ is the identity matrix. Then, the CRLB of the parameters of interest $\xi$ can be expressed as

$$
\begin{aligned}
\operatorname{CRLB}(\boldsymbol{\delta}) & =\left[\mathbf{J}_{\delta \boldsymbol{\delta}}-\mathbf{J}_{\boldsymbol{\alpha} \delta} \mathbf{J}_{\boldsymbol{\alpha} \alpha}^{-1} \mathbf{J}_{\boldsymbol{\alpha} \delta}^{H}\right]^{-1} \\
& =\frac{N}{8 \pi^{2}\left\|\alpha_{2}\right\|^{2} M S N R}\left[\begin{array}{ll}
\left(\left(B_{\mathrm{rms}}^{2}+f_{c}^{2}\right) \varepsilon_{1}-\frac{f_{c}^{2} \varepsilon_{4}^{2}}{M N}\right) \cos ^{2} \varphi_{1} & -\left(\left(B_{\mathrm{rms}}^{2}+f_{c}^{2}\right) \varepsilon_{3}-\frac{f_{c}^{2} \varepsilon_{4} \varepsilon_{5}}{M N}\right) \sin \varphi_{1} \cos \varphi_{1} \\
-\left(\left(B_{\mathrm{rms}}^{2}+f_{c}^{2}\right) \varepsilon_{3}-\frac{f_{c}^{2} \varepsilon_{4} \varepsilon_{5}}{M N}\right) \sin \varphi_{1} \cos \varphi_{1}\left(\left(B_{\mathrm{rms}}^{2}+f_{c}^{2}\right) \varepsilon_{2}-\frac{f_{c}^{2} \varepsilon_{5}^{2}}{M N}\right) \sin ^{2} \varphi_{1}
\end{array}\right]^{-1}
\end{aligned}
$$

According to the Smith's criterion, the 2D SARLs in this case are

$$
\begin{aligned}
& \delta_{\varphi}=\sqrt{\operatorname{CRLB}(\varphi)}=\sqrt{[\operatorname{CRLB}(\boldsymbol{\delta})]_{11}}, \\
& \delta_{\phi}=\sqrt{\operatorname{CRLB}(\phi)}=\sqrt{[\operatorname{CRLB}(\boldsymbol{\delta})]_{22}} .
\end{aligned}
$$

As is shown in (28), the derivation of SARL in closed form is difficult in the context of MIMO radar. However, in the case of UCA, $\varepsilon_{1}=\varepsilon_{2}=M N\left(r_{t}^{2}+r_{r}^{2}\right) /\left(2 c^{2}\right)$ and $\varepsilon_{3}=\varepsilon_{4}=\varepsilon_{5}=0$. Then, the CRLB can be simplified as

$$
\begin{aligned}
\operatorname{CRLB}(\boldsymbol{\delta})= & \frac{c^{2}}{4 \pi^{2}\left\|\alpha_{2}\right\|^{2} M^{2}\left\|\alpha_{2}\right\|^{2}\left(r_{t}^{2}+r_{r}^{2}\right)\left(B_{\mathrm{rms}}^{2}+f_{c}^{2}\right) \mathrm{SNR}} \\
& \times\left[\begin{array}{cc}
\frac{1}{\cos ^{2} \varphi_{1}} & 0 \\
0 & \frac{1}{\sin ^{2} \varphi_{1}}
\end{array}\right] .
\end{aligned}
$$

Consequently, the SARLs of elevation and azimuth are

$$
\begin{aligned}
& \delta_{\varphi}=\frac{c}{2 \pi M\left\|\alpha_{2}\right\| \cos \varphi_{1}} \sqrt{\frac{1}{\left(r_{t}^{2}+r_{r}^{2}\right)\left(f_{c}^{2}+B_{\mathrm{rms}}^{2}\right) \mathrm{SNR}}}, \\
& \delta_{\phi}=\frac{c}{2 \pi M\left\|\alpha_{2}\right\| \sin \varphi_{1}} \sqrt{\frac{1}{\left(r_{t}^{2}+r_{r}^{2}\right)\left(f_{c}^{2}+B_{\mathrm{rms}}^{2}\right) \mathrm{SNR}}} .
\end{aligned}
$$

4.3. Relationship between the SARL Based on DetectionTheoretic and Estimation-Theoretic Approaches. The SARLs have been derived based on the detection-theoretic and estimation-theoretic approaches, respectively. As described in [26], the noncentrality parameter $\lambda\left(p_{f a}, p_{d}\right)$ is given as

$$
\lambda\left(p_{f a}, p_{d}\right)=(\boldsymbol{\delta}-0)^{T}\left(\left[\mathbf{J}^{-1}(\boldsymbol{\xi})\right]_{\delta \delta}\right)^{-1}(\boldsymbol{\delta}-0),
$$

where $\left[J^{-1}(\xi)\right]_{\delta \delta}$ denotes the $\mathbf{J}_{\delta \delta}$ defined in (26). As the observation time is sufficiently long, (33) is equivalent to

$$
\begin{aligned}
\lambda\left(p_{f a}, p_{d}\right) & \cong(\boldsymbol{\delta}-0)^{T}\left(\left.\left[\mathbf{J}^{-1}(\boldsymbol{\xi})\right]_{\delta \boldsymbol{\delta}}\right|_{\boldsymbol{\delta}=\mathbf{0}}\right)^{-1}(\boldsymbol{\delta}-0) \\
& =\boldsymbol{\delta}^{T}\left(\left.\operatorname{CRLB}(\boldsymbol{\delta})\right|_{\boldsymbol{\delta}=\mathbf{0}}\right)^{-1} \boldsymbol{\delta},
\end{aligned}
$$

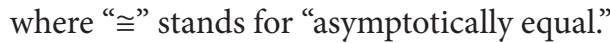

The conclusion in (34) can be verified by (16) and (28). Moreover, $\sqrt{\lambda\left(p_{f a}, p_{d}\right)}$, the proportionality constant corresponding to a given couple $\left(P_{f a}, P_{d}\right)$, is called "translation factor" (from CRLB to the SRL) in [20]. Thus, the asymptotic SRL based on the hypothesis test approach is consistent with the SRL based on the CRLB approach (i.e., using the Smith's criterion). Furthermore, the SRL based on Smith's criterion is equivalent to an asymptotically UMP test among all invariant statistical tests when $\lambda\left(p_{f a}, p_{d}\right)=1$. Consequently, (34) shows that the detection-based approach is unified with the estimation-based approach [20].

\section{Discussion and Numerical Analysis}

The 2D SARLs for UWB MIMO noise radar have been derived in Sections 3 and 4. In this section, the results expressed in (16) and (28) are discussed. Then some numerical simulations are made to analysis the factors impacting the 2D SARLs. 
TABLE 1: RMS bandwidth for different signals.

\begin{tabular}{lcc}
\hline Types of spectra & Expression & RMS bandwidth \\
\hline $\begin{array}{l}\text { Rectangular } \\
\text { low-frequency } \\
\text { spectrum }\end{array}$ & $\rho(f)= \begin{cases}\rho_{0}, & |f| \leq B_{w} / 2 \\
0, & |f|>B_{w} / 2\end{cases}$ & $B_{w} / 2 \sqrt{3}$ \\
\hline $\begin{array}{l}\text { Gaussian } \\
\text { spectrum }\end{array}$ & $\rho(f)=\rho_{0} \exp \left(-2 f^{2} / \sigma^{2} B_{w}^{2}\right)$ & $\sigma B_{w} / 2$ \\
\hline
\end{tabular}

5.1. Discussion. The following comments are intended to provide further insight into the results obtained in Sections 3 and 4.

(A1) Since the SARL is inversely proportional to RMS bandwidth $B_{\text {rms }}$ and carrier frequency $f_{c}$, increasing $B_{\text {rms }}$ and $f_{c}$ will improve the SARL, while for narrowband signals, $B_{\mathrm{rms}} / f_{c} \ll 1$, the SARL is inversely proportional to $f_{c}$ and independent of $B_{\text {rms }}$. As $B_{\text {rms }}$ is the integration over the range of frequencies with nonzero signal content, $B_{\mathrm{rms}}$ is determined by both the bandwidth $B_{w}$ and the distribution of PSD. As is shown in Table 1, the RMS bandwidth of noise waveforms for different distributions of PSD is different, which leads to different SARL.

(A2) As is shown in (28), the off-diagonal elements of the $2 \times 2$-order $\operatorname{CRLB}(\boldsymbol{\delta})$ corresponding to $\delta_{\varphi}$ and $\delta_{\phi}$ are not equivalent to zero, which means the CRLB is coupled and the SARL of one direction parameter $\left(\delta_{\varphi}\right.$ or $\left.\delta_{\phi}\right)$ is degraded by the uncertainty of another. The uncoupling is important which means that the SARL of one parameter is independent of others [28]. Since both diagonal and off-diagonal elements of $\mathbf{J}_{\boldsymbol{\delta} \boldsymbol{\delta}}$ depend on the array geometry, the optimum array geometry can be found to get the best resolution limit. As the off-diagonal elements of $\mathbf{J}_{\delta \delta}$ are zero, the array geometry for monostatic MIMO radar with UCA configuration satisfies the uncoupling conditions in [1].

(A3) The SARL is strongly reliant on the relative geometry of antennas versus target location. This dependency is incorporated in the terms of $\phi_{1}$ and $\varphi_{1}$. Moreover, it is apparent from (28) that there is a tradeoff between the SARLs of the azimuth and elevation. For example, a set of antennas' locations that increases the SARL of azimuth may result in a low SARL of elevation, as we expect intuitively. This is caused by the fact that $\delta_{\phi}$ is summation of sine functions and $\delta_{\varphi}$ is summation of cosine functions. In order to truly determine the minimum achievable SARLs in both azimuth and elevation, we need to minimize the overall SARL, defined as the total SARL in the next part.

(A4) As the SARLs in (16) and (28) are achieved for the coherent processing approach, the phase information is used which leads to the resolution limit improvement comparing with the noncoherent processing approach. However, both time synchronization and phase synchronization between the transmitters and receivers are necessary in this case, while only time synchronization is necessary in the noncoherent case [7], which is a challenge for the radar system.

(A5) The SARL, as expressed by CRLB, provides a tight bound at high SNR, while the CRLB is not tight at low-SNR case; thus, a more rigid bound is necessary for the estimation accuracy in this case, which is beyond this paper.

5.2. Numerical Simulation. The factors, impacting the $2 \mathrm{D}$ SARLs of MIMO radar with UWB noise waveforms, are analyzed by numerical simulations as follows. The transmit signals are set up as ideal bandlimited Gaussian noise waveforms with $f_{c}=9 \mathrm{GHz}$ and $B_{w}=0.2 f_{c}$; then, the RMS bandwidth is $B_{\mathrm{rms}}=B_{w} / 2 \sqrt{3}$. And we consider the case that the number of antennas is $M=N=4$ and all antennas lie on a disk of radius $r_{t}=r_{r}=r=1 \mathrm{~m}$.

First, the SARLs for different target directions and array geometry configurations are simulated to verify the comments A2 and A3. Assume that the transmitters and receivers are configured in the same array geometry. Consider four relative geometries of antennas versus target location in Figure 2, where " $x$ " represents the target location with the azimuth $\phi_{1}$ from the vertical view.

It can be shown from Figure 3 that the SARL is strongly related to array geometry and target location. As for UCA in type (d), $\varepsilon_{3}=\varepsilon_{4}=\varepsilon_{5}=0$ satisfies the uncoupling conditions described in [1], while the array geometries described in Figures 2(a) 2(c) are coupling configurations. As discussed above, the coupling increases the parameter uncertainty which degrades the SARL, and the conclusion is verified in Figure 3. Meanwhile, the aperture size of the array for type (d) is bigger than the other types of array geometry. Furthermore, comparing Figure 3(a) with Figure 3(b), the SARL of elevation degrades with the elevation of target, while the SARL of azimuth is enhanced by the increasing elevation. The contradiction makes it hard to design the array geometry versus the target direction.

As described in [20], the SARL (in the two-target case) is asymptotically proportional to the square root of the lower bound of the MSAE (in the single-target case). The MSAE is defined as

$$
\operatorname{MSAE}_{\mathrm{CRLB}} \triangleq \sin ^{2} \varphi \operatorname{CRLB}(\phi)+\operatorname{CRLB}(\varphi),
$$

where $\operatorname{CRLB}(\phi)$ and $\operatorname{CRLB}(\varphi)$ are the CRLBs on $\phi$ and $\varphi$ (in the single-target case, $\phi=\phi_{1}=\phi_{2}$ and $\varphi=\varphi_{1}=$ $\varphi_{2}$ ), respectively. $\sqrt{\mathrm{MSAE}_{\mathrm{CRLB}}}$ contains the CRLBs of the azimuth and elevation and provides an overall measure of the resolution limit; thus, $\sqrt{\mathrm{MSAE}_{\mathrm{CRLB}}}$ can be used as the "total SARL." Moreover, it is also a very useful quality measure for gaining physical insight into the SARL in $3 \mathrm{D}$ space. Figure 4 shows that $\sqrt{\mathrm{MSAE}_{\mathrm{CRLB}}}$ varies with the elevation and azimuth of target for different types of array geometry. There are some lowest points in each subfigure, which can be chosen as the tradeoff between the SARLs of azimuth and elevation in certain applications. And the tradeoff elevation and azimuth differ for different array geometry configurations. When designing the radar system, the radar plane can be adjusted to 


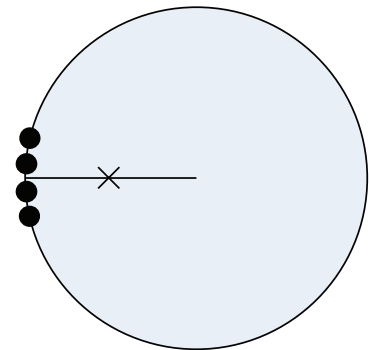

Antenna $\times$ Target

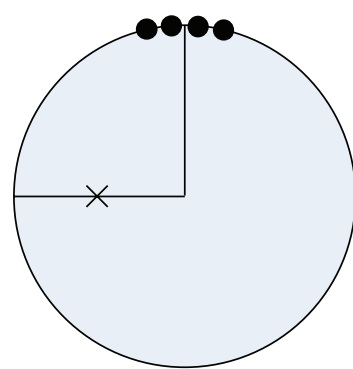

- Antenna $\times$ Target

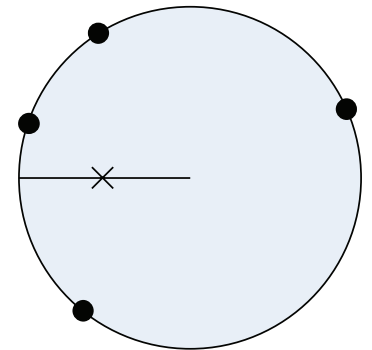

Antenna $\times$ Target

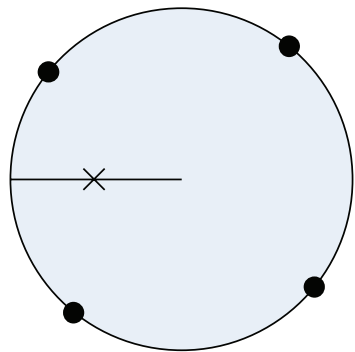

Antenna $\times$ Target

(d)

(a)

(b)

(c)

FIGURE 2: Different types of antenna geometry. (a) All elements concentrated at locations $r e^{j \phi_{1}}$ or $r e^{j\left(\phi_{1}+\pi\right)}$. (b) All elements concentrated at
locations $r e^{j\left(\phi_{1}+\pi / 2\right)}$ or $r e^{j\left(\phi_{1}-\pi / 2\right)}$. (c) The elements are randomly located at the disk. (d) The elements are uniformly located on the disk.

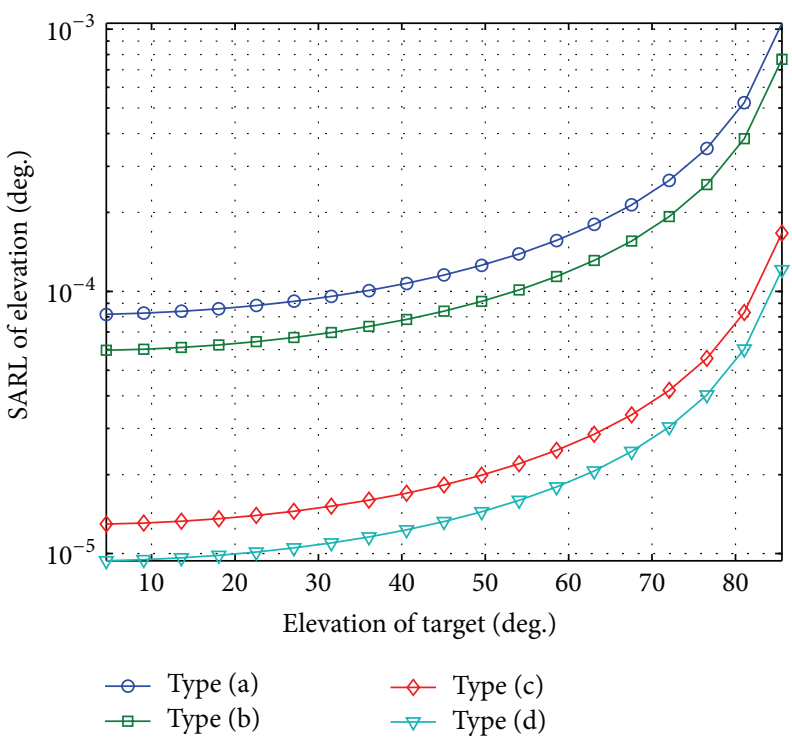

(a) SARL of elevation versus the elevation of target

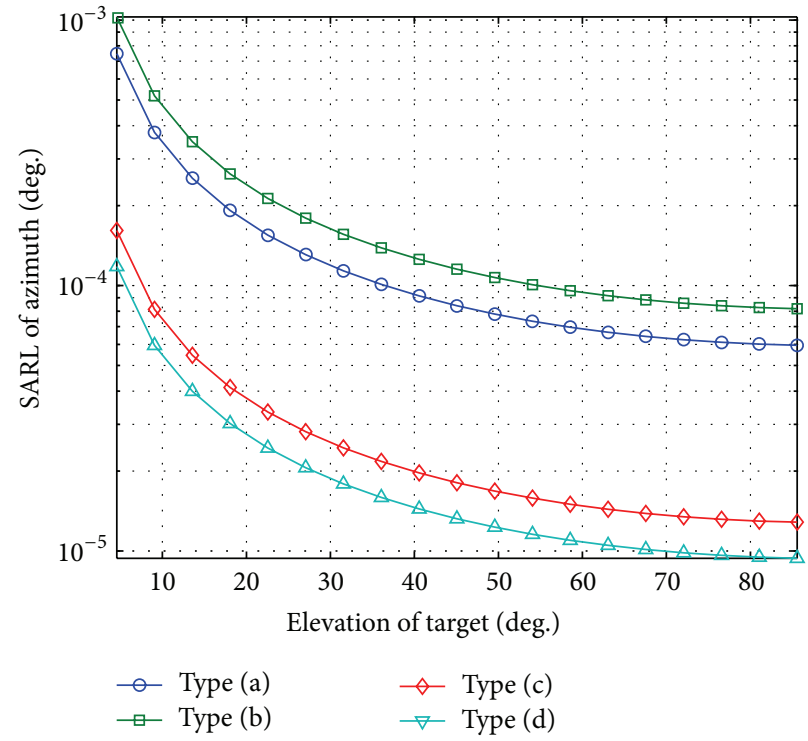

(b) SARL of azimuth versus the elevation of target

FIGURE 3: SARLs of elevation and azimuth versus the elevation of target for different types of array geometry.

make the target located at the tradeoff elevation and azimuth from the plane, which will lead to the best SARL.

Next, we focus on the effects of other factors on the SARL. In the simulations, both the transmitters and receivers are assumed to be arranged in UCA, and the SARL based on detection approach is considered.

The effect of RCS on the resolution limit can be concluded directly from (16) and (28). The SARL is improved as the RCS increases. Although RCS does not affect the transmit waveform, the received signal power and the probability of detection increase as the RCS increases, which induces a better resolution limit. Thus the target characteristic indeed could affect the resolution limit which is ignored by the conventional radar resolution theory. In the framework of our derivations, target $\mathrm{T} 1$ is assumed as the reference target with a known direction and RCS, while target T2 is unknown and is close to T1; thus, the SARL is independent of the RCS of T2. However, the RCS values of both targets could affect the SARL as the received signal power and SNR will increase if the RCS values increase. In our future work, we consider the resolution of two targets in case that both the targets are unknown although the derivations become complex. Some related work has been published in [29].

Figure 5 shows the effect of detection parameters where only $\delta_{\varphi}$ is considered (the same way is done for $\delta_{\phi}$ ). From Figure 5, the resolution limit decreases as $p_{d}$ increases and $p_{f a}$ decreases, since increasing $p_{d}$ and decreasing $p_{f a}$ lead to a more selective decision. Then the resolution limit can be improved by increasing $p_{f a}$ and calling for a lower $p_{d}$. However, the performance of detection will be depressed in this case. 


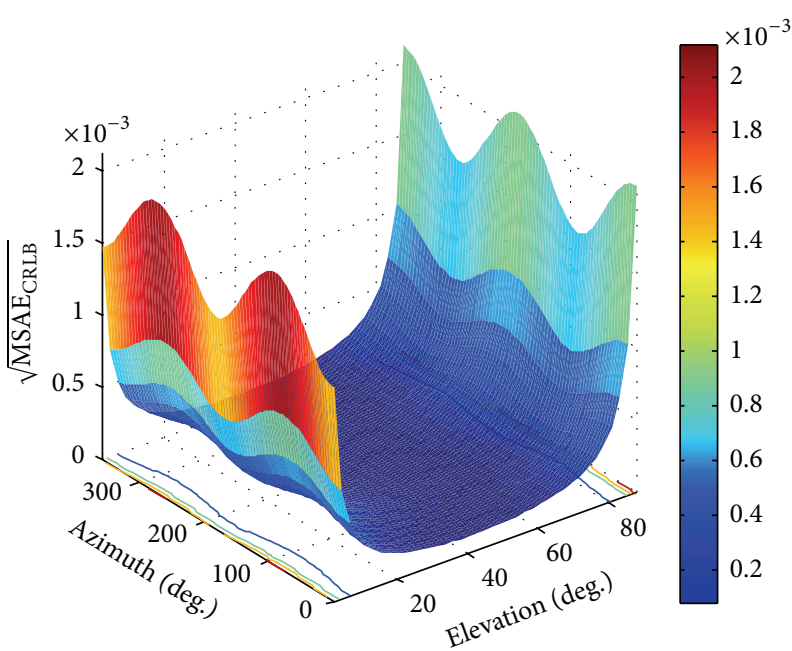

(a)

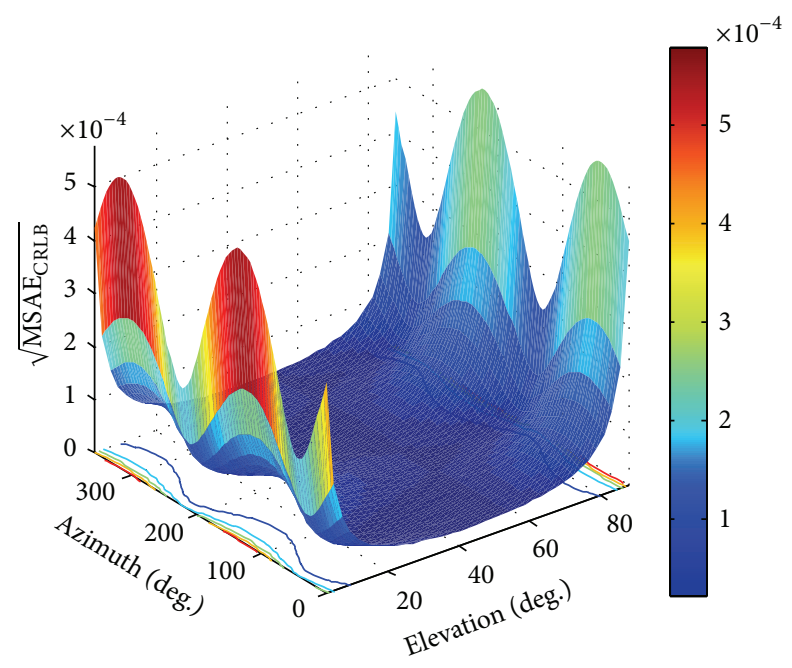

(c)

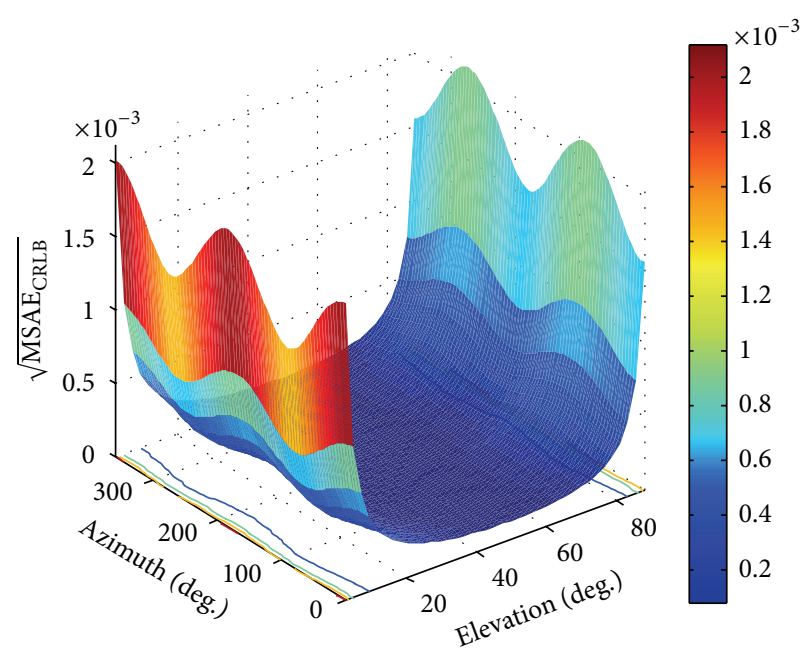

(b)

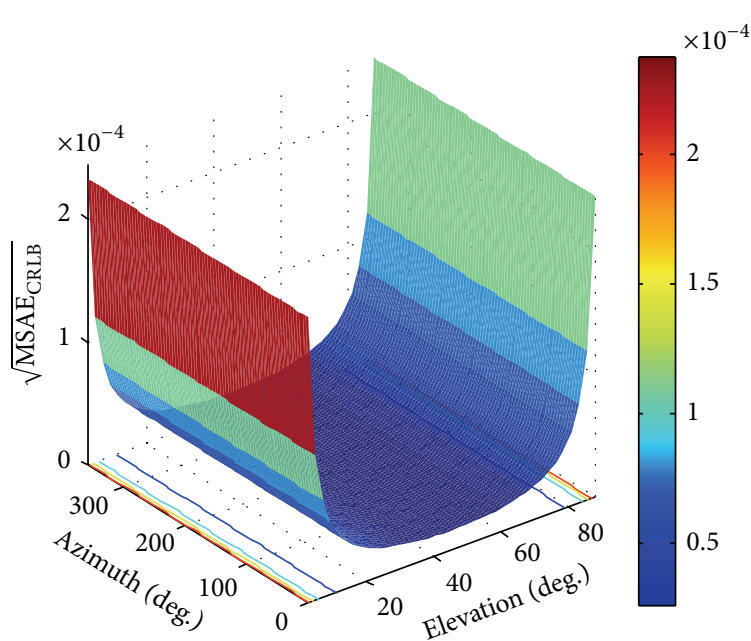

(d)

FIGURE 4: $\sqrt{\mathrm{MSAE}_{\mathrm{CRLB}}}$ versus the elevation and azimuth of target for different types of array geometry.

Figure 6 shows the resolution cells for different target directions and SNR with $\left(p_{f a}, p_{d}\right)=(0.01,0.99)$. For visual convenience, $\delta_{\varphi}$ and $\delta_{\phi}$ are scaled up to 120 times. Obviously, increasing SNR can improve the SARL. Meanwhile, the SARL varies with the target direction because of the spatial diversity of radar, which means that the SARL is "nonuniform" on the whole space. Moreover, the resolution cell is ellipse which means that the SARL varies at different directions for a given target location.

Consider the SARL of elevation $\delta_{\varphi}$ as shown in (31), the relationship between SARL and the radius of the arrays is simulated in Figure 7. The simulation parameters are configured as $M=N=4, r_{t}=r_{r}=r \in\{1 \mathrm{~m}, 2 \mathrm{~m}, 3 \mathrm{~m}\}$, and $\varphi_{1}=1^{\circ}$. Figure 7 represents that increasing the radius of the arrays will lead to the resolution limit improvement, since a lager virtual aperture is achieved in this case, according to the MIMO radar virtual aperture theory.

\section{Conclusion}

Resolution limit can be interpreted as the minimal difference to resolve two closely spaced targets and is a critical quantity to evaluate the performance of a radar system. In this paper, we have derived the explicit expressions for 2D SARLs of elevation and azimuth for UWB MIMO noise radar, which are defined using GLRT based on hypothesis test and estimation approach based on Smith's criterion, respectively. The relationship between the two approaches has been proved and verified. Meanwhile, we have analyzed the effects of SNR, detection parameters, transmit waveforms, and array geometry and the parameters of target on the SAR and some significant conclusions are achieved as follows. (1) The SARLs based on the detection and estimation approaches are unified. (2) The array geometry is an important factor that impacts the SARL. (3) There is a tradeoff between the SARLs of 


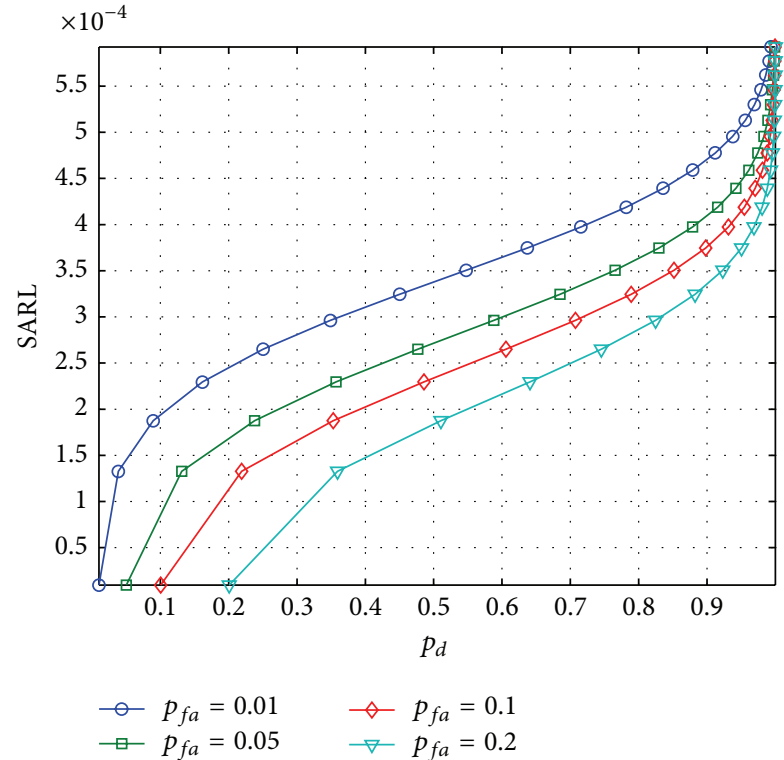

FIGURE 5: SARL as a function of detection parameters $\left(p_{f a}, p_{d}\right)$ for $\mathrm{SNR}=20 \mathrm{~dB}$ and $\varphi_{1}=1^{\circ}$.

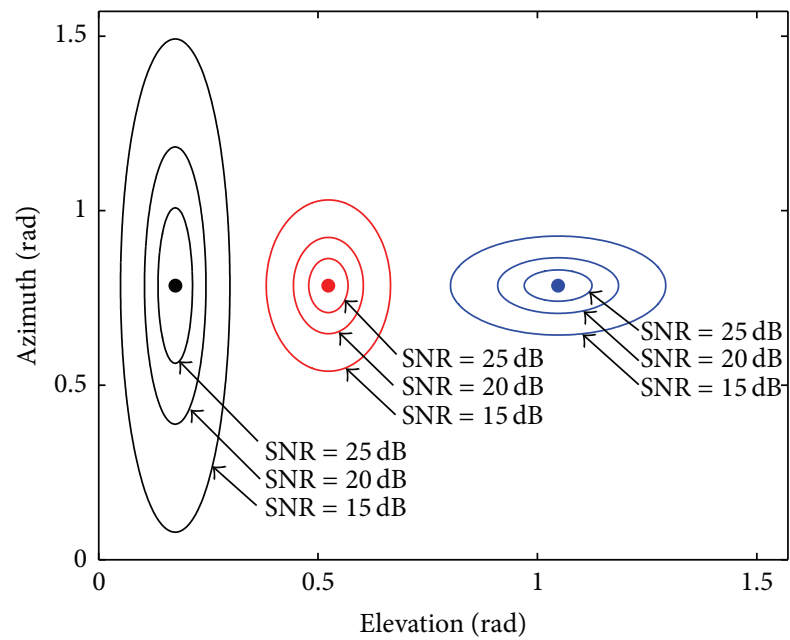

FIgURE 6: Resolution cell versus target direction for elevation $\varphi_{1} \epsilon$ $\left\{10^{\circ}, 30^{\circ}, 60^{\circ}\right\}$ and $\left(p_{f a}, p_{d}\right)=(0.01,0.99)$.

elevation and azimuth, which can be determined by the total SARL $\sqrt{M_{\text {SAE }} \text { CRLB }}$. (4) The SARLs of MIMO radar on the whole space and at different directions are "nonuniform". (5) The target characteristic and detection parameters (if the detection approach is used) can impact the resolution limit. (6) Increasing the bandwidth and SNR will result in the SARL improvement. Compared with the conventional radar resolution theory, the SRL reflects not only the effect of transmit waveforms, but also SNR, parameters of target, and measurement approach. Therefore, the SRL describes the practical resolution ability of radar effectively and provides the optimization criterion for radar system design.

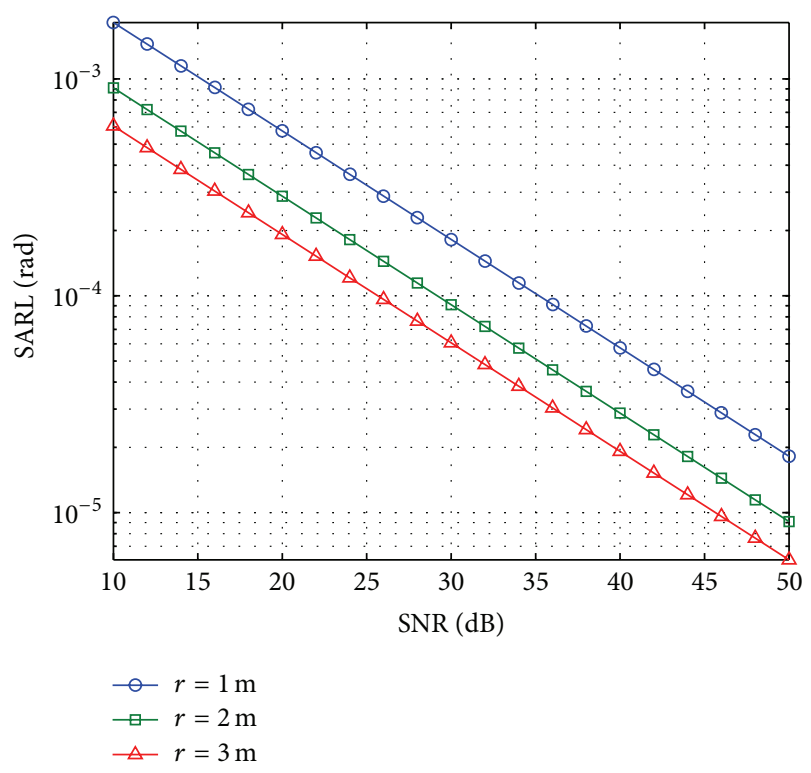

FIGURE 7: SARL as a function of $r$ and SNR (in dB).

\section{Appendix}

Derivation of the FIM in (20) is as follows.

The first derivative of $\log p(\mathbf{y} \mid \xi)$ with respect to the elements of $\boldsymbol{\xi}$ is

$$
\begin{aligned}
& \frac{\partial \log p(\mathbf{y} \mid \boldsymbol{\xi})}{\partial \alpha_{2}^{R}} \\
& =\frac{2}{\sigma_{n}^{2}} \sum_{m=1}^{M} \int_{T} \operatorname{Re}\left\{\left(y_{m}(t)-\sum_{l=1}^{2} \alpha_{l} \sum_{n=1}^{N} s_{n}\left(t-\tau_{m n}^{l}\right) e^{-j 2 \pi f_{c} \tau_{m n}^{l}}\right)\right. \\
& \left.\times\left(\sum_{n=1}^{N} s_{n}\left(t-\tau_{m n}^{2}\right) e^{-j 2 \pi f_{c} \tau_{m n}^{2}}\right)^{*}\right\} d t, \\
& \begin{aligned}
=\frac{2}{\sigma_{n}^{2}} \sum_{m=1}^{M} \int_{T} \operatorname{Re}\{( & \left.y_{m}(t)-\sum_{l=1}^{2} \alpha_{l} \sum_{n=1}^{N} s_{n}\left(t-\tau_{m n}^{l}\right) e^{-j 2 \pi f_{c} \tau_{m n}^{l}}\right) \\
& \left.\times\left(j \sum_{n=1}^{N} s_{n}\left(t-\tau_{m n}^{2}\right) e^{-j 2 \pi f_{c} \tau_{m n}^{2}}\right)^{*}\right\} d t,
\end{aligned}
\end{aligned}
$$

$$
\begin{aligned}
& \frac{\partial \log p(\mathbf{y} \mid \boldsymbol{\xi})}{\partial \delta_{\varphi}} \\
& =\frac{-2 \cos \varphi_{1}}{\sigma_{n}^{2}} \\
& \quad \times \sum_{m=1}^{M} \int_{T} \operatorname{Re} \\
& \quad \times\left\{\left(y_{m}(t)-\sum_{l=1}^{2} \alpha_{l} \sum_{n=1}^{N} s_{n}\left(t-\tau_{m n}^{l}\right) e^{-j 2 \pi f_{c} l_{m n}^{l}}\right)\right.
\end{aligned}
$$




$$
\begin{gathered}
\times\left(\alpha_{2} \sum_{n=1}^{N} a_{m n}\left(\dot{s}_{n}\left(t-\tau_{m n}^{2}\right)+j 2 \pi f_{c} s_{n}\left(t-\tau_{m n}^{2}\right)\right)\right. \\
\left.\left.\times e^{-j 2 \pi f_{c} \tau_{m n}^{2}}\right)^{*}\right\} d t
\end{gathered}
$$

$$
\begin{aligned}
& \frac{\partial \log p(\mathbf{y} \mid \boldsymbol{\xi})}{\partial \delta_{\phi}} \\
& =\frac{2 \sin \varphi_{1}}{\sigma_{n}^{2}} \\
& \quad \times \sum_{m=1}^{M} \int_{T} \operatorname{Re}\left\{\left(y_{m}(t)-\sum_{l=1}^{2} \alpha_{l} \sum_{n=1}^{N} s_{n}\left(t-\tau_{m n}^{l}\right) e^{-j 2 \pi f_{c} \tau_{m n}^{l}}\right)\right. \\
& \times\left(\alpha _ { 2 } \sum _ { n = 1 } ^ { N } b _ { m n } \left(\dot{s}_{n}\left(t-\tau_{m n}^{2}\right)\right.\right. \\
& \left.+j 2 \pi f_{c} s_{n}\left(t-\tau_{m n}^{2}\right)\right) \\
& \left.\left.\times e^{-j 2 \pi f_{c} \tau_{m n}^{2}}\right)^{*}\right\} d t .
\end{aligned}
$$

Denote snr $=\sigma_{s}^{2} / \sigma_{n}^{2}$ and use the conclusions in (24) and then apply the second derivative to (A.1) (A.4) to define FIM $\mathbf{J}(\boldsymbol{\xi})$ with the following elements:

$$
\begin{aligned}
{[\mathbf{J}(\boldsymbol{\xi})]_{11} } & =-\left.E_{\mathbf{y} \mid \xi}\left\{\frac{\partial^{2} \log p(\mathbf{y} \mid \boldsymbol{\xi})}{\left(\partial \alpha_{2}^{R}\right)^{2}}\right\}\right|_{\delta_{\varphi}=0, \delta_{\phi}=0} \\
& =2 M N \operatorname{snr}, \\
{[\mathbf{J}(\boldsymbol{\xi})]_{22} } & =-\left.E_{\mathbf{y} \mid \xi}\left\{\frac{\partial^{2} \log p(\mathbf{y} \mid \boldsymbol{\xi})}{\left(\partial \alpha_{2}^{I}\right)^{2}}\right\}\right|_{\delta_{\varphi}=0, \delta_{\phi}=0} \\
& =2 M N \operatorname{snr}, \\
{[\mathbf{J}(\boldsymbol{\xi})]_{21} } & =[\mathbf{J}(\boldsymbol{\xi})]_{12} \\
& =-\left.E_{\mathbf{y} \mid \xi}\left\{\frac{\partial^{2} \log p(\mathbf{y} \mid \boldsymbol{\xi})}{\partial \alpha_{2}^{R} \partial \alpha_{2}^{I}}\right\}\right|_{\delta_{\varphi}=0, \delta_{\phi}=0} \\
& =\frac{2}{\sigma_{n}^{2}} \operatorname{Re}\left\{-j M N \sigma_{s}^{2}\right\} \\
& =0,
\end{aligned}
$$$$
[\mathbf{J}(\boldsymbol{\xi})]_{31}=[\mathbf{J}(\boldsymbol{\xi})]_{13}
$$$$
=-\left.E_{\mathbf{y} \mid \xi}\left\{\frac{\partial^{2} \log p(\mathbf{y} \mid \boldsymbol{\xi})}{\partial \alpha_{2}^{R} \partial \delta_{\varphi}}\right\}\right|_{\delta_{\varphi}=0, \delta_{\phi}=0}
$$$$
=4 \pi f_{c} \alpha_{2} \operatorname{snr} \cos \varphi_{1} \varepsilon_{4},
$$

$[\mathbf{J}(\xi)]_{41}=[\mathbf{J}(\xi)]_{14}$

$$
\begin{aligned}
& =-\left.E_{\mathbf{y} \mid \xi}\left\{\frac{\partial^{2} \log p(\mathbf{y} \mid \boldsymbol{\xi})}{\partial \alpha_{2}^{R} \partial \delta_{\varphi}}\right\}\right|_{\delta_{\varphi}=0, \delta_{\phi}=0} \\
& =-4 \pi f_{c} \alpha_{2}^{I} \operatorname{snr} \sin \varphi_{1} \varepsilon_{5},
\end{aligned}
$$

$$
[\mathbf{J}(\boldsymbol{\xi})]_{32}=[\mathbf{J}(\boldsymbol{\xi})]_{23}
$$

$$
=-\left.E_{\mathbf{y} \mid \xi}\left\{\frac{\partial^{2} \log p(\mathbf{y} \mid \boldsymbol{\xi})}{\partial \alpha_{2}^{I} \partial \delta_{\varphi}}\right\}\right|_{\delta_{\varphi}=0, \delta_{\phi}=0}
$$

$$
=-4 \pi f_{c} \alpha_{2}^{R} \operatorname{snr} \cos \varphi_{1} \varepsilon_{4},
$$

$[\mathbf{J}(\boldsymbol{\xi})]_{42}=[\mathbf{J}(\boldsymbol{\xi})]_{24}$

$$
\begin{aligned}
& =-\left.E_{\mathbf{y} \mid \xi}\left\{\frac{\partial^{2} \log p(\mathbf{y} \mid \xi)}{\partial \alpha_{2}^{I} \partial \delta_{\phi}}\right\}\right|_{\delta_{\varphi}=0, \delta_{\phi}=0} \\
& =4 \pi f_{c} \alpha_{2}^{R} \operatorname{snr} \sin \varphi_{1} \varepsilon_{5},
\end{aligned}
$$

$$
\begin{aligned}
{[\mathbf{J}(\boldsymbol{\xi})]_{33} } & =-\left.E_{\mathbf{y} \mid \xi}\left\{\frac{\partial^{2} \log p(\mathbf{y} \mid \boldsymbol{\xi})}{\left(\partial \delta_{\varphi}\right)^{2}}\right\}\right|_{\delta_{\varphi}=0, \delta_{\phi}=0} \\
& =8 \pi^{2}\left\|\alpha_{2}\right\|^{2}\left(B_{\mathrm{rms}}^{2}+f_{c}^{2}\right) \operatorname{snr} \cos ^{2} \varphi_{1} \varepsilon_{1},
\end{aligned}
$$

$$
\begin{aligned}
{[\mathbf{J}(\boldsymbol{\xi})]_{44} } & =-\left.E_{\mathbf{y} \mid \boldsymbol{\xi}}\left\{\frac{\partial^{2} \log p(\mathbf{y} \mid \boldsymbol{\xi})}{\left(\partial \delta_{\phi}\right)^{2}}\right\}\right|_{\delta_{\varphi}=0, \delta_{\phi}=0} \\
& =8 \pi^{2}\left\|\alpha_{2}\right\|^{2}\left(B_{\mathrm{rms}}^{2}+f_{c}^{2}\right) \operatorname{snr} \sin ^{2} \varphi_{1} \varepsilon_{2}, \\
{[\mathbf{J}(\boldsymbol{\xi})]_{34} } & =[\mathbf{J}(\boldsymbol{\xi})]_{34} \\
& =-\left.E_{\mathbf{y} \mid \boldsymbol{\xi}}\left\{\frac{\partial^{2} \log p(\mathbf{y} \mid \boldsymbol{\xi})}{\partial \delta_{\varphi} \partial \delta_{\phi}}\right\}\right|_{\delta_{\varphi}=0, \delta_{\phi}=0} \\
& =-8 \pi^{2}\left\|\alpha_{2}\right\|^{2}\left(B_{\mathrm{rms}}^{2}+f_{c}^{2}\right) \operatorname{snr} \sin \varphi_{1} \cos \varphi_{1} \varepsilon_{3} .
\end{aligned}
$$

\section{Conflict of Interests}

The authors declare that there is no conflict of interests regarding the publication of this paper.

\section{Acknowledgments}

This work was supported by the National Natural Science Foundation of China (no. 61302149 and no. 61101182) and the Research Fund for the Doctoral Program of Higher Education of China (20124307110013). The authors would like to thank the editors and reviewers for their insightful comments. 


\section{References}

[1] H. Chen, X. Li, H. Wang, and Z. Zhuang, "Performance bounds of direction finding and its applications for multiple-input multiple-output radar," IET Radar, Sonar \& Navigation, vol. 8, no. 3, pp. 251-263, 2014.

[2] J. Li and P. Stoica, Eds., MIMO Radar Signal Processing, John Wiley \& Sons, New York, NY, USA, 2008.

[3] W. J. Chen and R. M. Narayanan, "Comparison of the estimation performance of coherent and non-coherent ambiguity functions for an ultrawideband multi-input-multi-output noise radar," IET Radar, Sonar and Navigation, vol. 6, no. 1, pp. 49-59, 2012.

[4] W.-J. Chen and R. M. Narayanan, "CGLRT plus TDL beamforming for ultrawideband MIMO noise radar," IEEE Transactions on Aerospace and Electronic Systems, vol. 48, no. 3, pp. 1858-1869, 2012.

[5] W.-J. Chen and R. M. Narayanan, "Antenna placement for minimizing target localization error in UWB MIMO noise radar," IEEE Antennas and Wireless Propagation Letters, vol. 10, pp. 135-138, 2011.

[6] M. Dawood and R. M. Narayanan, "Generalised wideband ambiguity function of a coherent ultrawideband random noise radar," IEE Proceedings: Radar, Sonar and Navigation, vol. 150, no. 5, pp. 379-386, 2003.

[7] H. Godrich, A. M. Haimovich, and R. S. Blum, "Target localization accuracy gain in MIMO radar-based systems," IEEE Transactions on Information Theory, vol. 56, no. 6, pp. 27832803, 2010.

[8] P. Woodward, Probability and Information Theory: With Applications to Radar, Pergamon, New York, NY, USA, 1957.

[9] J. M. Speiser, "Wideband ambiguity functions," IEEE Transactions on Information Theory, vol. 13, no. 1, pp. 122-123, 1967.

[10] H. Yan, G. Shen, R. Zetik, O. Hirsch, and R. S. Thoma, "Ultrawideband MIMO ambiguity function and its factorability," IEEE Transactions on Geoscience and Remote Sensing, vol. 51, no. 1, pp. 504-519, 2013.

[11] G. S. Antonio, D. R. Fuhrmann, and F. C. Robey, "MIMO radar ambiguity functions," IEEE Journal on Selected Topics in Signal Processing, vol. 1, no. 1, pp. 167-177, 2007.

[12] Y. I. Abramovich and G. J. Frazer, "Bounds on the volume and height distributions for the MIMO radar ambiguity function," IEEE Signal Processing Letters, vol. 15, pp. 505-508, 2008.

[13] S. T. Smith, "Statistical resolution limits and the complexified Cramér-Rao bound," IEEE Transactions on Signal Processing, vol. 53, no. 5, pp. 1597-1609, 2005.

[14] H. Cox, "Resolving power and sensitivity to mismatch of optimum array processors," Journal of the Acoustical Society of America, vol. 54, no. 3, pp. 771-785, 1973.

[15] M. N. El Korso, R. Boyer, A. Renaux, and S. Marcos, "Statistical resolution limit for the multidimensional harmonic retrieval model- hypothesis test and Cramér-Rao bound approaches," EURASIP Journal on Advances in Signal Processing, vol. 2011, article 12, 2011.

[16] M. Shahram and P. Milanfar, "Statistical and informationtheoretic analysis of resolution in imaging," IEEE Transactions on Information Theory, vol. 52, no. 8, pp. 3411-3437, 2006.

[17] M. N. El Korso, R. Boyer, A. Renaux, and S. Marcos, "On the asymptotic resolvability of two point sources in known subspace interference using a GLRT-based framework," Signal Processing, vol. 92, no. 10, pp. 2471-2483, 2012.
[18] A. Amar and A. J. Weiss, "Fundamental limitations on the resolution of deterministic signals," IEEE Transactions on Signal Processing, vol. 56, no. 11, pp. 5309-5318, 2008.

[19] M. Shahram and P. Milanfar, "On the resolvability of sinusoids with nearby frequencies in the presence of noise," IEEE Transactions on Signal Processing, vol. 53, no. 7, pp. 2579-2588, 2005.

[20] Z. Liu and A. Nehorai, "Statistical angular resolution limit for point sources," IEEE Transactions on Signal Processing, vol. 55, no. 11, pp. 5521-5527, 2007.

[21] M. N. El Korso, R. Boyer, A. Renaux, and S. Marcos, "Statistical resolution limit for source localization with clutter interference in a MIMO radar context," IEEE Transactions on Signal Processing, vol. 60, no. 2, pp. 987-992, 2012.

[22] N. D. Tran, R. Boyer, S. Marcos, and P. Larzabal, "On the angular resolution limit for array processing in the presence of modeling errors," IEEE Transactions on Signal Processing, vol. 61, no. 19, pp. 4701-4706, 2013.

[23] M. N. El Korso, R. Boyer, A. Renaux, and S. Marcos, "Statistical resolution limit of the uniform linear cocentered orthogonal loop and dipole array," IEEE Transactions on Signal Processing, vol. 59, no. 1, pp. 425-431, 2011.

[24] R. Boyer, "Performance bounds and angular resolution limit for the moving colocated MIMO radar," IEEE Transactions on Signal Processing, vol. 59, no. 4, pp. 1539-1552, 2011.

[25] H. B. Lee, "The Cramer-Rao bound on frequency estimates of signals closely spaced in frequency," IEEE Transactions on Signal Processing, vol. 40, no. 6, pp. 1508-1517, 1992.

[26] S. M. Kay, Fundamentals of Statistical Signal Processing: Volume II: Detection Theory, chapter 6, Prentice-Hall, Englewood Cliffs, NJ, USA, 1993.

[27] D. C. Bell and R. M. Narayanan, "Theoretical aspects of radar imaging using stochastic waveforms," IEEE Transactions on Signal Processing, vol. 49, no. 2, pp. 394-400, 2001.

[28] M. Hawkes, "Effects of sensor placement on acoustic vectorsensor array performance," IEEE Journal of Oceanic Engineering, vol. 24, no. 1, pp. 33-40, 1999.

[29] H. Chen, W. Zhou, J. Yang, Y. Peng, and X. Li, "Manifold studies on fundamental limits of direction-finding multipleinput multiple-output radar systems," IET Radar, Sonar and Navigation, vol. 6, no. 8, pp. 708-718, 2012. 

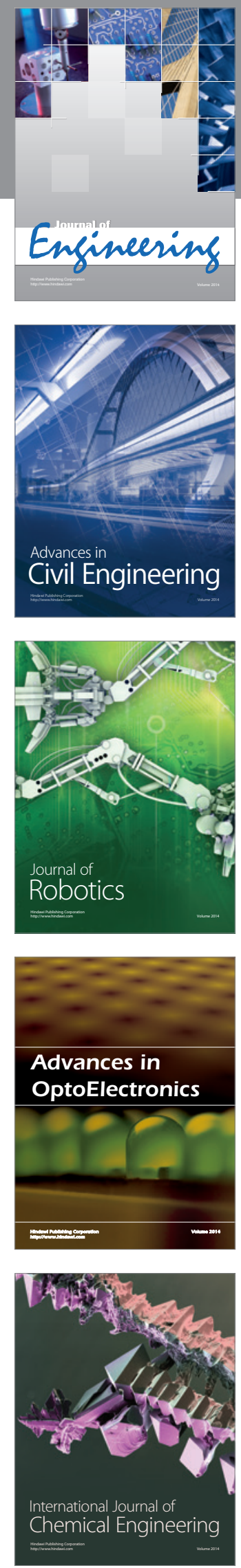

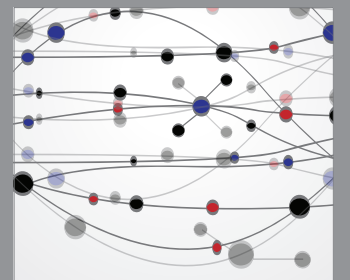

The Scientific World Journal
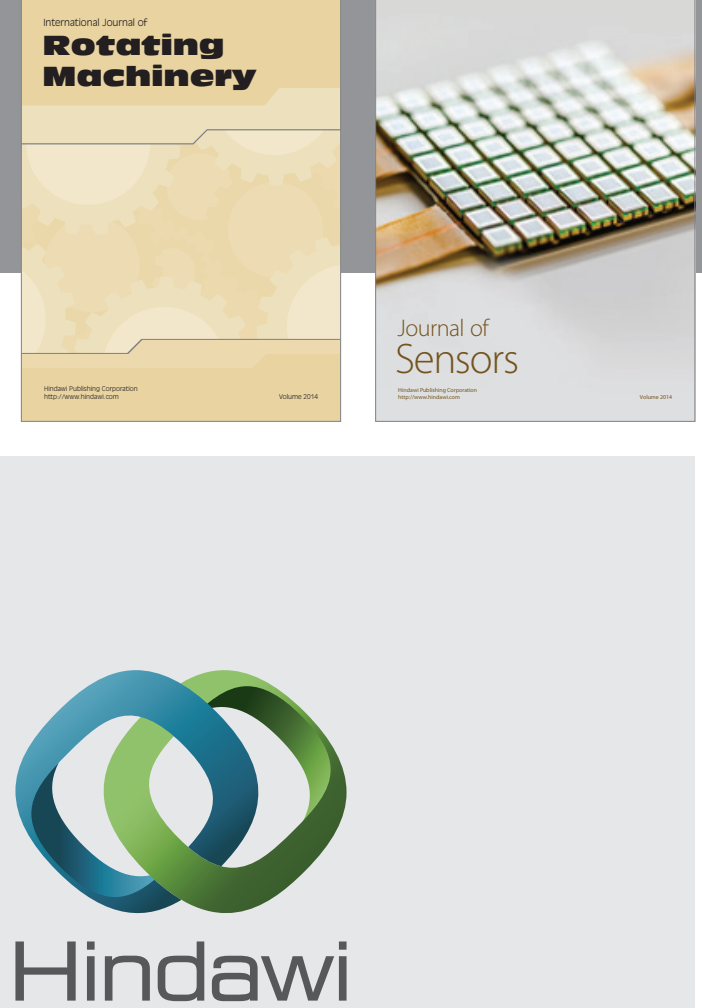

Submit your manuscripts at http://www.hindawi.com
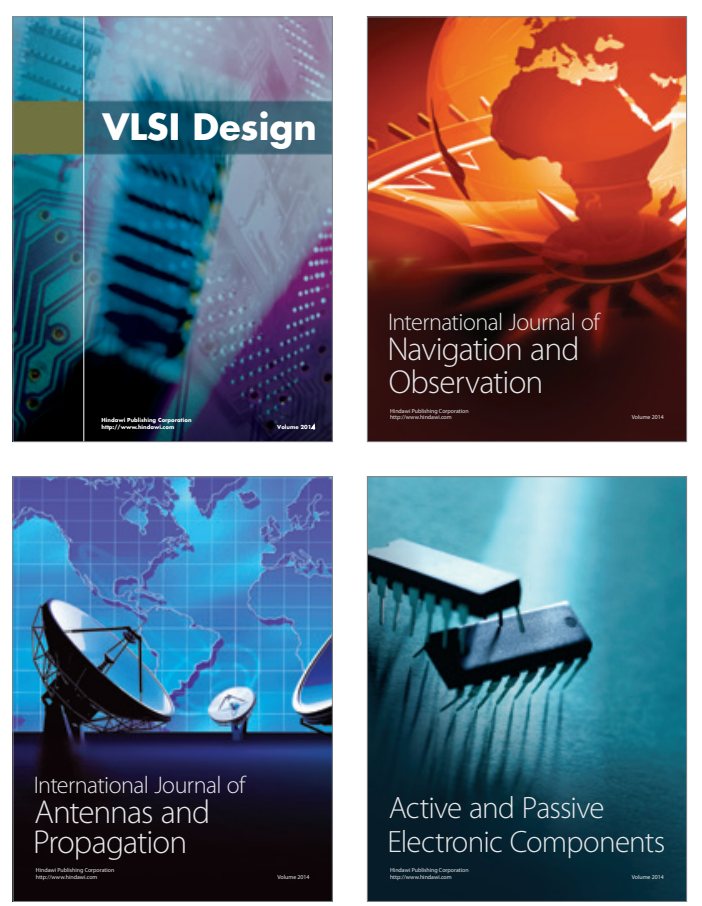
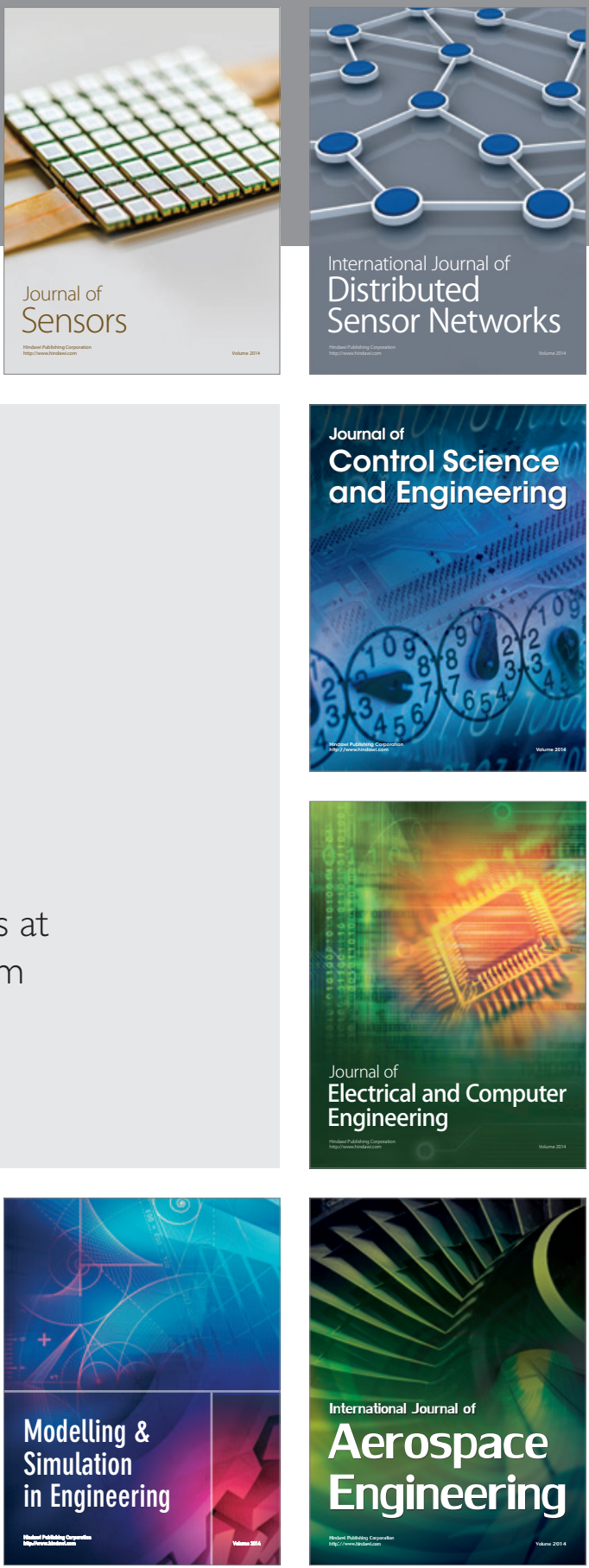

Journal of

Control Science

and Engineering
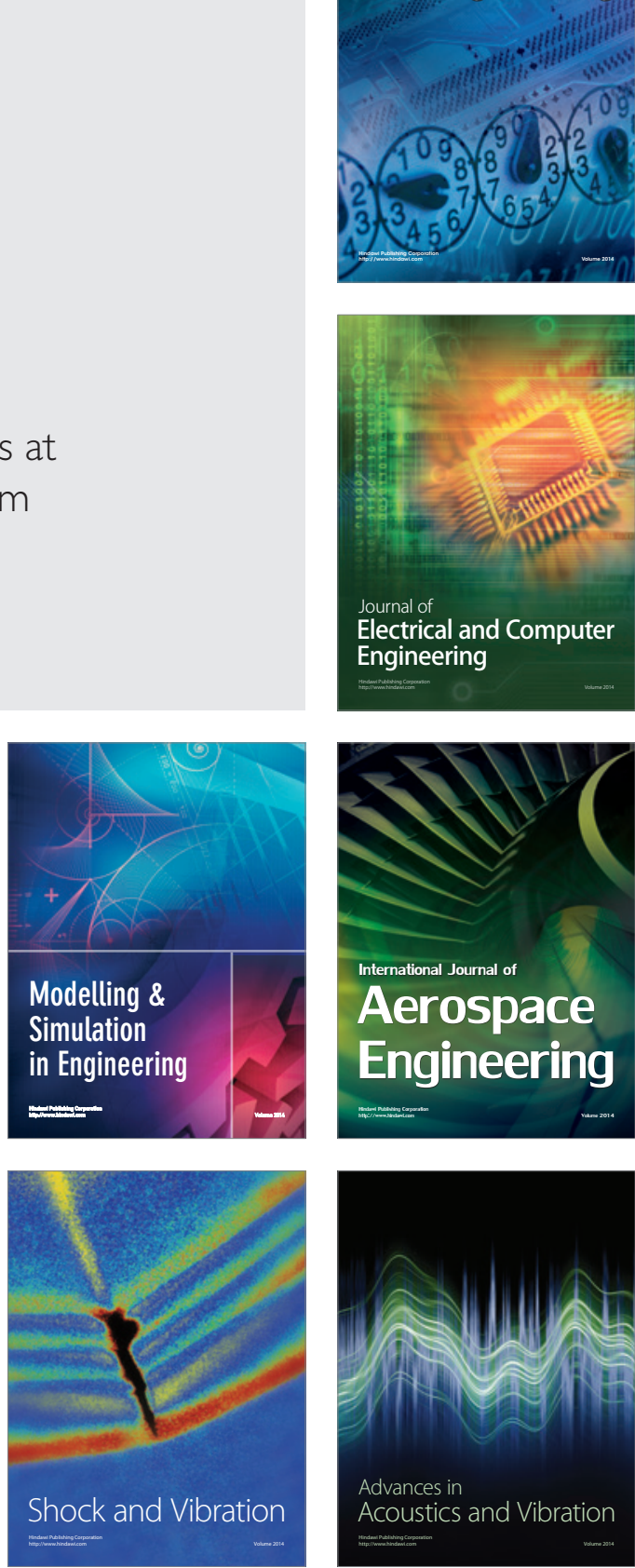